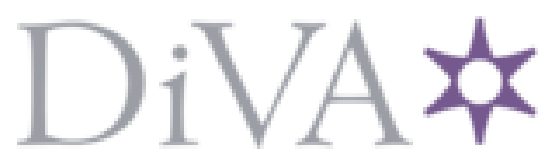

http://www.diva-portal.org

\title{
Postprint
}

This is the accepted version of a paper published in Footwear Science. This paper has been peerreviewed but does not include the final publisher proof-corrections or journal pagination.

Citation for the original published paper (version of record):

Fischer, K M., Willwacher, S., Arndt, A., Wolf, P., Brueggemann, G-P. (2017)

Calcaneal adduction in slow running: three case studies using intracortical pins..

Footwear Science, 9(2): 87-93

https://doi.org/10.1080/19424280.2017.1342704

Access to the published version may require subscription.

N.B. When citing this work, cite the original published paper.

Permanent link to this version:

http://urn.kb.se/resolve?urn=urn:nbn:se:gih:diva-4999 


\section{Calcaneal adduction in slow running: Three case studies using intracortical pins}

Katina Mira Fischer ${ }^{\text {a,b }}$, Steffen Willwacher ${ }^{\mathrm{a}, \mathrm{b}}$, Anton Arndt ${ }^{\mathrm{c}, \mathrm{d}}$, Peter Wolf ${ }^{\mathrm{e}}$, Gert - Peter

Brüggemann ${ }^{\mathrm{a}, \mathrm{b}}$

a Institute of Biomechanics and Orthopaedics, German Sport University, Cologne, Germany

${ }^{b}$ Institute of Functional Diagnostics, Cologne, Germany

${ }^{\mathrm{c}}$ The Swedish School of Sport and Health Sciences, Stockholm, Sweden

${ }^{\mathrm{d}}$ Karolinska Institute, Department CLINTEC, Stockholm, Sweden

e Sensory-Motor Systems Lab, ETH Zurich, Switzerland

\section{Corresponding Author}

Katina Mira Fischer

Institute of Biomechanics and Orthopaedics

German Sport University Cologne

Am Sportpark Müngersdorf 6

50933 Cologne, Germany

Phone: +49 22149827660

Fax: +492214971598

Email: k.fischer@dshs-koeln.de 
Dr. Steffen Willwacher

Institute of Biomechanics and Orthopaedics

German Sport University Cologne

Am Sportpark Müngersdorf 6

50933 Cologne, Germany

Phone: +49 22149827660

Fax: +49 2214971598

Email: s.willwacher@dshs-koeln.de

Prof. Dr. Anton Arndt

The Swedish School of Sport and Health Sciences

Box 5626 S-114 86 Stockholm, Sweden

and

Karolinska Institute, Department CLINTEC

SE-171 76 Stockholm, Sweden

Email: toni.arndt@gih.se

Dr. Peter Wolf

Sensory-Motor Systems Lab

ETH Zurich

Tannenstrasse 1

8092 Zurich, Switzerland

Email: peter.wolf@hest.ethz.ch

Prof. Dr. Gert-Peter Brüggemann

Institute of Biomechanics and Orthopaedics

Am Sportpark Müngersdorf 6

German Sport University Cologne

50933 Cologne, Germany

Phone: +49221 49825660

Fax: +49 2214971598

Email: brueggemann@dshs-koeln.de

Key Words: Foot Mechanics; Running; Calcaneal Adduction; Tibial Rotation; Intracortical Pins 


\title{
Calcaneal adduction in slow running: Three case studies using intracortical pins
}

\begin{abstract}
The aim of this study was to use bone anchored-markers to determine the bone movement of calcaneal adduction, eversion and tibial rotation in a global coordinate system and to describe the relationship of calcaneal adduction to tibial rotation. Furthermore, the amount of overall intra-foot motion in the transverse plane (metatarsal I relative to calcaneus) and its relationship to calcaneal adduction were quantified. Three male participants were assessed during slow running. A 10-camera motion analysis system was used for kinematic data capture of global bone orientations in 3D space for all bones of the foot and ankle complex. For the description of intrinsic articulations within the foot, the skeletal motion relative to the adjacent proximal segment in the transverse plane was calculated. Furthermore, the time of occurrence of maximum values was determined. The findings showed that calcaneal adduction of all participants amounted to $7.8 \pm 4.8^{\circ}$, which exceeded the magnitude of calcaneal eversion $\left(4.7 \pm 3.1^{\circ}\right)$. Although the inter-participant variability was high, considerable overall intra-foot motion in the transverse plane of the metatarsal I relative to the calcaneus was found to be $4.7 \pm 4.6^{\circ}$ and could be qualitatively related to calcaneal adduction. The present data provide evidence that next to calcaneal eversion, calcaneal adduction seems related to tibial rotation. Furthermore, overall intra-foot motion in the transverse plane seems related to calcaneal adduction. Controlling calcaneal adduction and overall intra-foot motion in the transverse plane may be a mechanism to control excessive tibial rotation in runners who suffer from overuse knee injuries. These findings could be used to provide an additional approach for future motion-control footwear design to control rearfoot adduction or overall within-foot motion.
\end{abstract}


Key Words: Foot Mechanics; Running; Calcaneal Adduction; Tibial Rotation; Intracortical Pins

\section{Introduction}

The knee joint is the most common site of overuse injuries in running with an incidence of up to $50 \%$ of all lower extremity injuries reported (van Gent et al., 2007). The risk of sustaining an injury has been associated with excessive internal tibial rotation among other risk factors. As a consequence of anatomical conditions at the ankle-joint complex, rearfoot eversion is coupled with internal tibial rotation (Tiberio, 1987). This movement transfer has been considered relevant in the etiology of several overuse knee injuries in runners. There are indications that excessive tibial rotation affects the patellar tendon tension direction and therefore, the patellofemoral contact pressure (McClay \& Manal, 1997) as well as friction of the iliotibial band (Hamill, Miller, Noehren, \& Davis, 2008; Noehren, Davis, \& Hamill, 2007). Consequently, footwear has been designed to counteract excessive foot and ankle motions by using strategies such as arch support, and midsole materials that differ in hardness. However, movement coupling at the anklejoint complex to a certain extent plays an important role in human locomotion and is harmless. Only if an individually higher extent of ankle-joint motion occurs, higher joint loading can be a consequence and may result in overuse injuries.

Previous research has provided descriptions of kinematic coupling from the foot to shank (DeLeo, Dierks, Ferber, \& Davis, 2004; McClay \& Manal, 1997; Stacoff, Nigg, Reinschmidt, van den Bogert, \& Lundberg, 2000; Stacoff, Nigg, Reinschmidt, van den Bogert, Lundberg, et al., 2000; Stacoff, Reinschmidt, et al., 2000). These studies have focused on the mechanical coupling between rearfoot eversion and tibial rotation neglecting potential coupling mechanisms of rearfoot adduction (rearfoot movement in the transverse plane) to tibial rotation (Figure 1). 
Nonetheless, considerable amounts of rearfoot motion within the transverse plane have been demonstrated (Cornwall \& McPoil, 1999; Hunt, Smith, Torode, \& Keenan, 2001; A. Leardini, Benedetti, Catani, Simoncini, \& Giannini, 1999; Moseley, Smith, Hunt, \& Gant, 1996; Rattanaprasert, Smith, Sullivan, \& Gilleard, 1999) in addition to rearfoot eversion and have recently been related to the amount of tibial rotation in running (Fischer, Willwacher, Hamill, \& Brüggemann, 2017). However, research on the relationship between rearfoot adduction and tibial rotation is limited and definitions of coupling mechanisms at the ankle-joint complex are therefore incomplete.

\section{[Figure 1 insert here]}

With the use of skin-mounted markers, recent in vivo research showed that both rearfoot adduction as well as rearfoot eversion measured in a global coordinate system were significantly correlated to tibial rotation in running (Fischer et al., 2017). Skin-mounted marker-based analyses offer the benefit of being non-invasive, thereby allowing for large sample sizes to be studied and avoiding the possibility of clinical complications. Nonetheless, bone-pin marker-based approaches enable a more precise measurement of the kinematics of individual bones, which are too small for valid marker placement or are inaccessible by surface markers. In addition, bonepin marker-based analyses are not affected by soft-tissue and movement artefacts (Alberto Leardini, Chiari, Croce, \& Cappozzo, 2005). To precisely understand the underlying mechanisms in the aetiology of knee pain, the amount of skeletal movement within the ankle joint and foot needs to be determined accurately, which in this regard favours bone pins over skin markers.

In addition to ankle-joint coupling mechanisms, it has been suggested that forefoot-rearfoot coupling can indirectly contribute to the amount of tibial rotation by increasing the amount of 
calcaneal motion (Fischer et al., 2017). Numerous biomechanical studies have examined intrafoot motion (Arndt et al., 2007; Damavandi, Dixon, \& Pearsall, 2010; Dixon, Tisseyre, Damavandi, \& Pearsall, 2011; Lundgren et al., 2008; Wolf et al., 2008) and its relationship to tibial rotation (Eslami, Begon, Farahpour, \& Allard, 2007; Fischer et al., 2017). However, to our best knowledge, only one study has determined the transverse intra-foot motion - transversal expansion (TE) in the context of movement transfer from rearfoot to tibia (Fischer et al., 2017). It has been shown that TE is directly related to higher rearfoot movement amplitudes in the transverse plane, which is related to tibial rotation (Fischer et al., 2017). However, for a detailed understanding, the present results need to be confirmed by means of a bone anchored marker approach to precisely describe the actual skeletal motion inside the foot and ankle-joint complex. Therefore, the purposes of the current study were to: (1) determine the excursion of calcaneal adduction and to observe its relationship to tibial rotation within a global coordinate system by means of bone anchored markers during running; (2) identify the amount of TE and its relation to calcaneal adduction; and (3) analyse intra-foot motion and its contribution to TE.

We hypothesised that: (1) there is a considerable amount of calcaneal adduction/abduction in running which is related to tibial rotation; (2) TE is related to calcaneal adduction.

The outcome of this study might have a direct impact on insole and running shoe design, especially with respect to arch supporting and motion-control elements.

\section{Methods}

To analyse the amount of global calcaneal transverse plane motion, as well as intra-foot motion occurring during running, a data-set of the in vivo study by Arndt et al. (2007) was reanalysed. 


\section{Participants and preparation}

Data of three healthy male volunteers (participant A, B and C; age: 52, 33 and 28 years; body mass: 88,110 and $71 \mathrm{~kg}$; height: $1.83,1.82$ and $1.80 \mathrm{~m}$; respectively) were included in the analysis. The study was approved by the local ethical committee of Huddinge University Hospital, Sweden. Intracortical pins were inserted by an experienced orthopaedic surgeon under local anaesthesia into nine separate bones (tibia, fibula, calcaneus, talus, navicular, cuboid, cuneiform I and metatarsal I and V), and a reflective marker triad was attached to each. For this study, information of only six bones (tibia, calcaneus [Calc], talus [Tal], navicular [Nav], cuneiform I [Cun I] and metatarsal I [Met I]) were included in the data analysis.

\section{Experiment}

Participants ran barefoot at a self-selected speed and cadence along a $9.5 \mathrm{~m}$ walkway. The running speed of participants $\mathrm{A}, \mathrm{B}$, and $\mathrm{C}$ was $2.1,1.9$ and $2.2 \mathrm{~m} / \mathrm{s}$, respectively, which was quite slow due to the restricted laboratory space. Data from the right foot were collected at $240 \mathrm{~Hz}$ using a 10-camera Pro Reflex optoelectric system (Qualysis, Göteborg, Sweden). Six to eight running trials per participant could be included in the data analysis. To determine the anatomical reference coordinate systems, the longitudinal axis of the foot was aligned with the anteroposterior axis of the global coordinate system by using a standing reference trial.

\section{Analysis}

Marker coordinates were filtered using a recursive fourth-order digital Butterworth filter (cut-off frequency, $10 \mathrm{~Hz}$ ). A mathematical optimisation of marker triad coordinates was performed for 
each marker triad to comply better with rigid body assumptions (Soderkvist \& Wedin, 1993). Local coordinate systems of each bone were aligned with the laboratory coordinate system in a standing reference trial. In the dynamic situation, the orientation of the local coordinate systems was determined by tracking the technical (marker triad-based) coordinate systems and combined with the rotational offset between the local and technical coordinate systems from the reference measurement (Cappozzo, Della Croce, Leardini, \& Chiari, 2005).

Global bone orientations in 3D space for all bones of the ankle-foot complex were determined for each running trial and then averaged for each participant during the stance phase of running, using the Cardan angle convention. The sequence of rotation was flexion-extension, adductionabduction, and internal-external rotation (Zatsiorsky, 1998).

Unlike previous studies, the orientation of bones was calculated in reference to the laboratory coordinate system. This approach was applied for all angular motions except for within-foot motion, which was described as the relative movement between bones. The global orientation approach was necessary in order to correctly describe the interrelationship or coupling of bony motions within the same plane of movement. If, for example, the distal and proximal bone motions within the transverse plane are perfectly coupled with each other (coupling coefficient $=$ 1), the classical joint-angle approach, which describes the orientation of the distal bone relative to the proximal, would indicate zero motion of the distal segment. As the movement coupling between two segments is classically derived from a least squares fit between the motions of the distal and proximal segments, zero motion of the distal segment would result in non-meaningful coupling coefficients. In order to describe the coupling of movement within the same plane of 
movement, it is indispensable to quantify the orientation of bones in the global (laboratory) coordinate system.

In order to extract information regarding the timing of bone movement, the time of maximum internal tibial rotation (T-TibRotMax) was determined during each stance phase. The global range of motion (RoM) values for the calcaneus in the frontal and transverse planes and for the tibia in the transverse plane in the range from touchdown to T-TibRotMax were calculated. The time at which the maximum values occurred was represented as a percentage of the stance phase (Max \% SP) for all three segments.

In addition, we determined the overall intra-foot motion in the transverse plane, defined as the movement of metatarsal I relative to the calcaneus, which will be termed TE in the context of this paper. During each stance phase, the time of maximum TE (T-TEMax) was determined.

For a better understanding of how the transverse plane intra-foot motion was distributed between the individual joints of the foot, RoMs in the transverse plane were calculated for the relative motion of Met I to the Calc, Tal to the Calc, Nav to the Tal, Cun I to the Nav and Met I to Cun I, using the Cardan angle convention and the same rotation sequence as for global orientation calculations. RoM values for these intra-foot motion variables were calculated in the range from touchdown to T-TEMax.

Because of the small sample size, no statistical tests were performed. Further details of the experimental procedures are described in Arndt et al. (2007). 


\section{Results}

Global RoM for calcaneal adduction was greater than eversion for two of three participants (Table 1, Figure 2). Inter-individual variability was high with means ranging between $4.9^{\circ}$ and $13.3^{\circ}$ for calcaneal adduction compared to $1.4^{\circ}$ and $7.5^{\circ}$ for calcaneal eversion (Table 1 , Figure 2). Participant $\mathrm{C}$ showed the highest RoM values for calcaneal adduction, eversion and internal tibial rotation. By comparing the Max $\% \mathrm{SP}$, the participant $\mathrm{C}$ also showed the best temporal correspondence of all maximum values (Table 1, Figure 2).

\section{[Table 1, Figure 2 insert here]}

The TE differed substantially between the participants, with values of $0.9^{\circ}$ (participant $\mathrm{B}$ ), $3.4^{\circ}$ (participant A) and $9.8^{\circ}$ (participant C) (Table 2, Figure 3). The participant $\mathrm{C}$ also displayed the highest calcaneal adduction $\left(13.3^{\circ}\right)$ (Table 1) with the best temporal correspondence of both maximum values (Figure 3).

\section{[Table 2, Figure 3 insert here]}

The contribution of relative segmental movement within the foot to the TE differed substantially between participants (Table 2, Figure 4). When qualitatively observing the results in all participants, the segmental movement of the navicular bone relative to the talus appeared to be related to the TE (Figure 4). As with the navicular relative to the talus, the metatarsal I moved considerably relative to the calcaneus, with the best temporal correspondence of the maximum values observed in participants A (Nav-Tal: 2.2 ${ }^{\circ}$; Met I-Cal: $\left.3.4^{\circ}\right)$ and C (Nav-Tal: $8.8^{\circ}$; Met ICal: $9.8^{\circ}$ ) (Table 2, Figure 4). Furthermore, in participant A, the calcaneus relative to the talus $\left(7.2^{\circ}\right)$ and in participant $\mathrm{C}$, the navicular relative to the cuneiform I $\left(3.8^{\circ}\right)$ contributed to an increasing TE (Table 2, Figure 4). 


\section{[Figure 4 insert here]}

\section{Discussion}

The purpose of this study was to use bone-anchored external markers to describe the actual bone movement of calcaneal adduction, eversion and tibial rotation in a global coordinate system and to observe the relationship of calcaneal adduction to tibial rotation. Furthermore, the amount of overall intra-foot motion in the transverse plane (metatarsal I relative to calcaneus) was analysed and its relationship to calcaneal adduction was determined. In addition, intra-foot motion and its contribution to TE were analysed.

It was hypothesised that a considerable amount of calcaneal adduction exists in slow running. The findings of this bone pin-based approach showed that the mean $( \pm \mathrm{SD})$ calcaneal adduction of all participants amounts to $7.8 \pm 4.8^{\circ}$, which is higher than the magnitude of calcaneal eversion $\left(4.7 \pm 3.1^{\circ}\right)$. However, the variability of RoM values was high between the participants, with participant $\mathrm{C}$ showing the highest amounts for calcaneal adduction, eversion and internal tibial rotation. In contrast, participant A and B displayed similar magnitudes of RoM values with means ranging between $5.0 \pm 0.3^{\circ}, 3.3 \pm 2.8^{\circ}$ and $8.7 \pm 0.4^{\circ}$ for calcaneal adduction, eversion and internal tibial rotation respectively. This inter-participant variability indicates biological variability between people as a function of foot morphology, biomechanical properties of passive structures or muscle activation patterns.

Previous studies using skin markers have shown that global rearfoot adduction amounts to $6.1 \pm$ $2.7^{\circ}$ and rearfoot eversion to $8.0 \pm 4.4^{\circ}$ during barefoot running (Fischer et al., 2017). These results are in the same order of magnitude as the values found in the present study. A likely explanation for the slight discrepancy in values for frontal plane rearfoot motion was that the 
present data are from slow-running participants with speeds ranging between 1.9 and $2.2 \mathrm{~m} / \mathrm{s}$, as compared to a mean running speed of $3.5 \mathrm{~m} / \mathrm{s}$ in the study of Fischer et al. (2017). Furthermore, participants of both studies were running on different surfaces (laboratory surface vs. EVA foam [55 Shore C]). Studies analysing the effect of surface conditions on leg geometry have shown that foot-strike patterns varied with different degrees of hardness and compositions of surfaces to avoid pressure peaks underneath the heel (De Wit, De Clercq, \& Aerts, 2000; Willwacher, Regniet, Fischer, Oberländer, \& Brüggemann, 2014).

In contrast to previous studies which have examined the coupling of movements in different planes (e.g. eversion to internal tibial rotation), we applied a global bone-orientation approach. This was because the relationship between bones moving within the same plane of motion cannot be determined correctly using the classical joint-angle approach, where the movement of the distal segment is expressed relative to the proximal one. A joint-angle method may even result in deceptive results. Let us consider for example the relative movement between rearfoot adduction and internal tibial rotation. If the amount of internal tibial rotation is greater than calcaneal adduction, a relative abduction of the calcaneus would be determined by means of the joint-angle approach even if, in a global coordinate system, the calcaneus would be actually adducting. This would lead to a complete misinterpretation of the motion of the rearfoot and the actual relation between the two segments. Therefore, it is essential to use a global movement approach when addressing within-plane movement coupling.

The second purpose of this study was to identify the amount of TE and its relation to calcaneal adduction. The mean TE $\left(4.7 \pm 4.6^{\circ}\right.$; Calc-Met I) in the present study was smaller than the value reported by Lundgren et al. (2008), who reported an RoM of $14.7 \pm 5.3^{\circ}$ between the first 
metatarsal and talus during walking using intracortical pins. The differences may be attributed to the fact that different modes of locomotion and motion of different segments have been analysed. In accordance to the current results using the same segments and coordinate system conventions, Fischer et al. (2017) displayed a transverse plane intra-foot motion of $3.3 \pm 3.0^{\circ}$ by means of an on-skin marker approach. Despite the small number of participants and the high inter-participant variability in the current study, a good consistency of data determined with intracortical pins compared to an on-skin marker approach could be found, indicating that skin markers represent a non-invasive approximation to accurately determine TE.

In addition, a previous study comparing bone versus skin markers could not find a systematic over- or underestimation of movement by either of the two approaches (Nester et al., 2007), which supports this assumption.

It was expected that higher $\mathrm{TE}$ is related to greater calcaneal adduction, which would be consequently related to tibial rotation. Participant $\mathrm{C}$ who showed the highest RoM values for TE as well as for calcaneal adduction also demonstrated the best temporal correspondence of both maximum values. By analysing the contribution of relative bone movement to the TE, the variability between the participants was high. For all participants, a positive contribution to the TE could be observed only for the relative movement between the navicular and talus. Both participants A and C showed similar RoM values for the talus relative to the navicular and TE. Participant B displayed higher RoM values for the talus relative to the navicular than for TE. The inter-participant variability of intra-foot motion provides additional evidence that small effects of shoe-sole modifications on tibiocalcaneal movement reported in literature (Stacoff et al., 2001), may be due to inhomogeneous samples and the problem of neglecting individual characteristics 
by analysing central tendencies within groups. Based on these results, it is assumed that controlling for TE and therefore rearfoot adduction may have an additional effect on ankle-joint coupling, as calcaneal adduction is significantly correlated to internal tibial rotation (Fischer et al., 2017). Due to the orientation of the subtalar joint axis, there is evidence that both rearfoot eversion and rearfoot adduction occur together (Cornwall \& McPoil, 1999; Hunt et al., 2001; A. Leardini et al., 1999). By means of a partial linear correlation analysis Fischer et al. (2017) provided evidence that rearfoot adduction was significantly related to tibial rotation, while also controlling for rearfoot eversion. It can be assumed that despite the orientation of the subtalar joint axis, other factors influence calcaneal adduction and eversion. Therefore, both movements must be considered separately of each other.

We can speculate that traditional interventions (orthoses or shoe designs) focusing on controlling calcaneal eversion should be-reconsidered and that implementing designs to restrict TE and therefore rearfoot adduction may have an additional effect on ankle-joint coupling mechanisms. In this attempt, different approaches would be conceivable. One approach could be to simulate passive elastic structures of stiffer feet by implementing material into the shoe upper at the medial aspect of the shoe. Another approach could be to decouple the rear- from the forefoot element and to incorporate elements, using passive elastic structures with higher stiffness at the medial aspect and less stiff elements at the lateral aspect of the sole to restrict the TE in participants with a strong coupling to internal tibial rotation.

In running, calcaneal movement includes not only eversion/inversion but also adduction/abduction. To independently analyse the contribution of both movements to ankle-joint coupling mechanisms and therefore to tibial rotation, future research should address the effect of 
calcaneal adduction/abduction and eversion/inversion on tibial rotation by inducing both movements in an isolated manner. By means of a cadaver study, the relationship of calcaneal adduction on tibial rotation could be observed, without interference of calcaneal movement in the other two planes.

Nonetheless, a possible limitation of this study is of course its invasive character, and therefore the possibility of clinical complications, and the related limited number of available participants. It has been shown that the local anaesthesia did not significantly affect the subjects' kinematics (Maiwald et al., 2017). However, this bone pin-based approach permitted the precise measuring of individual bone kinematics, without interference of skin movement artefacts or soft-tissue vibrations. The precise measurement of the kinematic of individual bones was necessary to validate data of a previous study carried out by Fischer et al. (2017) using skin-mounted markers. In conclusion, this study has shown that both calcaneal eversion and calcaneal adduction occur in running. This result provides additional evidence that in addition to calcaneal eversion, calcaneal adduction seems related to tibial rotation. Furthermore, TE is related to calcaneal adduction, whereby the TE is subdivided into movements of distinct joints inside the foot. In all participants, only the relative movement between the navicular and talus contributed to an increasing TE, providing an approach for future motion-control footwear design.

\section{Disclosure statement}

There were no conflicts of interest with this study. 


\section{References}

Arndt, A., Wolf, P., Liu, A., Nester, C., Stacoff, A., Jones, R., Lundgren, P., \& Lundberg, A. (2007). Intrinsic foot kinematics measured in vivo during the stance phase of slow running. J Biomech, 40(12), 2672-2678

Cappozzo, A., Della Croce, U., Leardini, A., \& Chiari, L. (2005). Human movement analysis using stereophotogrammetry. Part 1: theoretical background. Gait \& Posture, 21(2), 186196. doi: 10.1016/j.gaitpost.2004.01.010

Cornwall, M. W., \& McPoil, T. G. (1999). Three-dimensional movement of the foot during the stance phase of walking. J Am Podiatr Med Assoc, 89(2), 56-66

Damavandi, M., Dixon, P. C., \& Pearsall, D. J. (2010). Kinematic adaptations of the hindfoot, forefoot, and hallux during cross-slope walking. Gait \& Posture, 32(3), 411-415

De Wit, B., De Clercq, D., \& Aerts, P. (2000). Biomechanical analysis of the stance phase during barefoot and shod running. J Biomech, 33(3), 269-278

DeLeo, A. T., Dierks, T. A., Ferber, R., \& Davis, I. S. (2004). Lower extremity joint coupling during running: a current update. Clinical Biomechanics, 19(10), 983-991.

Dixon, P. C., Tisseyre, M., Damavandi, M., \& Pearsall, D. J. (2011). Inter-segment foot kinematics during cross-slope running. Gait Posture, 33(4), 640-644. doi: 10.1016/j.gaitpost.2011.02.010

Eslami, M., Begon, M., Farahpour, N., \& Allard, P. (2007). Forefoot-rearfoot coupling patterns and tibial internal rotation during stance phase of barefoot versus shod running. Clin Biomech (Bristol, Avon), 22(1), 74-80

Fischer, K. M., Willwacher, S., Hamill, J., \& Brüggemann, G. P. (2017). Tibial rotation in running: Does rearfoot adduction matter? Gait \& Posture, 51, 188-193

Hamill, J., Miller, R., Noehren, B., \& Davis, I. (2008). A prospective study of iliotibial band strain in runners. Clin Biomech (Bristol, Avon), 23(8), 1018-1025.

Hunt, A. E., Smith, R. M., Torode, M., \& Keenan, A. M. (2001). Inter-segment foot motion and ground reaction forces over the stance phase of walking. Clin Biomech (Bristol, Avon), 16(7), 592-600

Leardini, A., Benedetti, M. G., Catani, F., Simoncini, L., \& Giannini, S. (1999). An anatomically based protocol for the description of foot segment kinematics during gait. Clin Biomech (Bristol, Avon), 14(8), 528-536

Leardini, A., Chiari, L., Croce, U. D., \& Cappozzo, A. (2005). Human movement analysis using stereophotogrammetry: Part 3. Soft tissue artifact assessment and compensation. Gait \& Posture, 21(2), 212-225. doi: http://dx.doi.org/10.1016/j.gaitpost.2004.05.002

Lundgren, P., Nester, C., Liu, A., Arndt, A., Jones, R., Stacoff, A., Wolf, P., \& Lundberg, A. (2008). Invasive in vivo measurement of rear-, mid- and forefoot motion during walking. Gait \& Posture, 28(1), 93-100. doi: http://dx.doi.org/10.1016/j.gaitpost.2007.10.009

Maiwald, C., Arndt, A., Nester, C., Jones, R., Lundberg, A., \& Wolf, P. (2017). The effect of intracortical bone pin application on kinetics and tibiocalcaneal kinematics of walking gait. Gait \& Posture, 52, 129-134

McClay, I., \& Manal, K. (1997). Coupling parameters in runners with normal and excessive pronation. Journal of Applied Biomechanics, 13, 109-124 
Moseley, L., Smith, R., Hunt, A., \& Gant, R. (1996). Three-dimensional kinematics of the rearfoot during the stance phase of walking in normal young adult males. Clin Biomech (Bristol, Avon), 11(1), 39-45

Nester, C., Jones, R. K., Liu, A., Howard, D., Lundberg, A., Arndt, A., Lundgren, P., Stacoff, A., \& Wolf, P. (2007). Foot kinematics during walking measured using bone and surface mounted markers. J Biomech

Noehren, B., Davis, I., \& Hamill, J. (2007). ASB clinical biomechanics award winner 2006 prospective study of the biomechanical factors associated with iliotibial band syndrome. Clinical Biomechanics, 22(9), 951-956

Rattanaprasert, U., Smith, R., Sullivan, M., \& Gilleard, W. (1999). Three-dimensional kinematics of the forefoot, rearfoot, and leg without the function of tibialis posterior in comparison with normals during stance phase of walking. Clin Biomech (Bristol, Avon), 14(1), 14-23

Soderkvist, I., \& Wedin, P. A. (1993). Determining the movements of the skeleton using wellconfigured markers. Journal of Biomechanics, 26(12), 1473-1477

Stacoff, A., Nigg, B. M., Reinschmidt, C., van den Bogert, A. J., \& Lundberg, A. (2000). Tibiocalcaneal kinematics of barefoot versus shod running. Journal of biomechanics, 33(11), 1387-1395

Stacoff, A., Nigg, B. M., Reinschmidt, C., van den Bogert, A. J., Lundberg, A., Stussi, E., \& Denoth, J. (2000). Movement coupling at the ankle during the stance phase of running. Foot Ankle Int, 21(3), 232-239

Stacoff, A., Reinschmidt, C., Nigg, B. M., van den Bogert, A. J., Lundberg, A., Denoth, J., \& Stussi, E. (2000). Effects of foot orthoses on skeletal motion during running. Clin Biomech (Bristol, Avon), 15(1), 54-64

Stacoff, A., Reinschmidt, C., Nigg, B. M., Van Den Bogert, A. J., Lundberg, A., Denoth, J., \& Stussi, E. (2001). Effects of shoe sole construction on skeletal motion during running. Med Sci Sports Exerc, 33(2), 311-319

Tiberio. (1987). The Effect of Excessive Subtalar Joint Pronation on Patellofemoral Mechanics: A Theoretical Model. Journal of Orthopaedic \& Sports Physical Therapy, 9(4), 160-165. doi: 10.2519/jospt.1987.9.4.160

van Gent, R. N., Siem, D., van Middelkoop, M., van Os, A. G., Bierma-Zeinstra, S. M., \& Koes, B. W. (2007). Incidence and determinants of lower extremity running injuries in long distance runners: a systematic review. Br J Sports Med, 41(8), 469-480; discussion 480.

Willwacher, S., Regniet, L., Fischer, K. M., Oberländer, K. D., \& Brüggemann, G.-P. (2014). The effect of shoes, surface conditions and sex on leg geometry at touchdown in habitually shod runners. Footwear Science, 6(3), 129-138

Wolf, P., Stacoff, A., Liu, A., Nester, C., Arndt, A., Lundberg, A., \& Stuessi, E. (2008).

Functional units of the human foot. Gait \& Posture, 28(3), 434-441. doi: http://dx.doi.org/10.1016/j.gaitpost.2008.02.004

Zatsiorsky, V. M. (1998). Kinematics of human motion: Human Kinetics. 
Table 1. Individual global mean (SD) RoM ranging from touchdown to T-TibRotMax and Max \% SP for calcaneal adduction, calcaneal eversion and internal tibial rotation.

\begin{tabular}{ccccccccc} 
& \multicolumn{2}{c}{ Calcaneal adduction $\left(^{\circ}\right)$} & & \multicolumn{2}{c}{ Calcaneal eversion $\left(^{\circ}\right)$} & & \multicolumn{2}{c}{ Internal tibial rotation $\left(^{\circ}\right)$} \\
\cline { 2 - 3 } Participant & Mean (SD) & Max \% SP & & Mean (SD) & Max \% SP & & Mean (SD) & Max \% SP \\
\hline A & $5.2^{\circ}(1.6)$ & 69 & & $5.3(1.2)$ & 43 & & $9.0(1.7)$ & 55 \\
B & $4.9(1.3)$ & 48 & & $1.4(0.7)$ & 45 & & $8.5(1.4)$ & 44 \\
C & $13.3(1.7)$ & 55 & & $7.5(1.4)$ & 52 & $18.8(0.8)$ & 54 \\
\hline
\end{tabular}

Table 2. Individual mean (SD) RoM of relative bone movement in the transverse plane described in the planes of the relevant proximal segment's coordinate system ranging from touchdown to T-TEMax.

\begin{tabular}{|c|c|c|c|c|c|}
\hline & Tal-calc & Nav-tal & Cun I-nav & Met I-cun I & Met I-calc \\
\hline Participant & Mean (SD) & Mean (SD) & Mean (SD) & Mean (SD) & Mean (SD) \\
\hline A & $7.2(0.5)$ & $2.2(1.4)$ & $-2.6(1.2)$ & $0.0(1.0)$ & $3.4(0.6)$ \\
\hline B & $-2.6(1.5)$ & $4.4(3.2)$ & $0.5(3.3)$ & $-1.1(0.9)$ & $0.9(0.5)$ \\
\hline $\mathrm{C}$ & $-1.0(1.4)$ & $8.8(1.2)$ & $3.8(1.2)$ & $-3.0(0.9)$ & $9.8(0.7)$ \\
\hline
\end{tabular}

\section{Figure descriptions}

Figure 1. Schematic presentation of rearfoot motion. (A) frontal plane movement and (B) transverse plane movement.

Figure 2. Individual global orientation curves for calcaneal adduction, calcaneal eversion and tibial rotation during the stance phase of running. The Max \% SP is indicated by the vertical lines.

Figure 3. Individual global orientation curves for calcaneal abduction (-)/adduction (+), as well as for transverse segmental movement of the metatarsal I relative to the calcaneus (+: abduction of the distal relative to the proximal segment) during the stance phase of running. The Max \% SP is indicated by the vertical lines.

Figure 4. Individual mean curves of relative bony movement in the transverse plane during the stance phase of running. Data are presented relative to the touchdown value of the respective angles. Positive values represent an abduction of the distal segment relative to the proximal segment. 
A

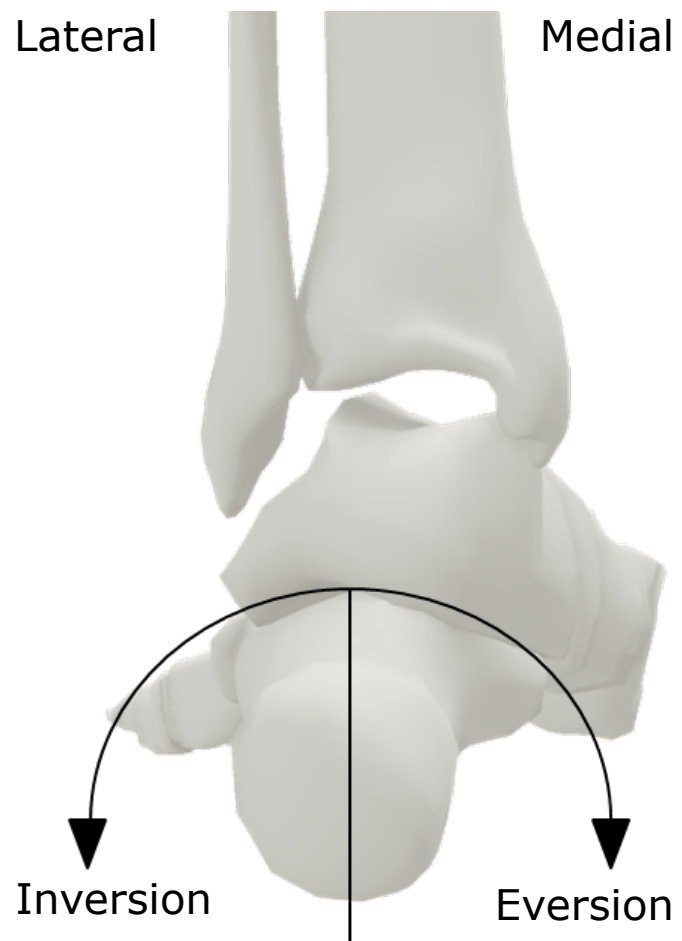

B

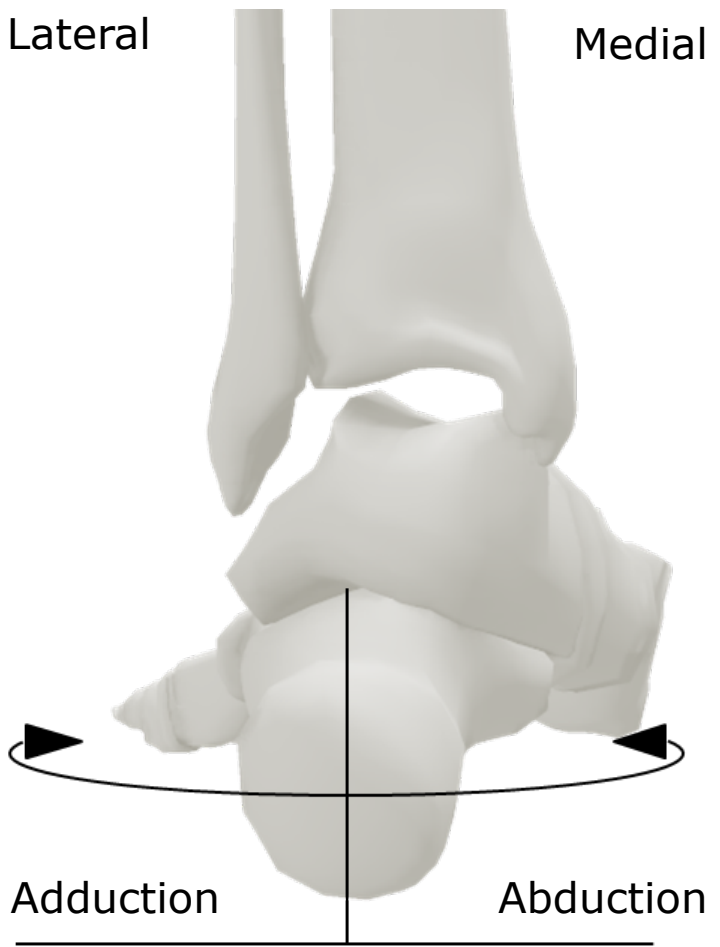

Figure 1 

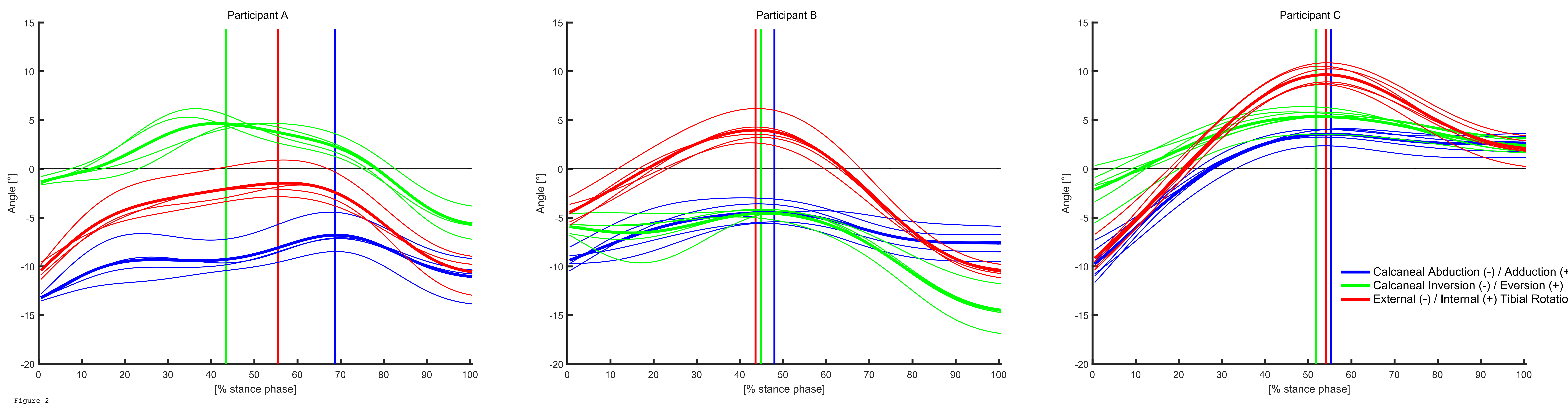

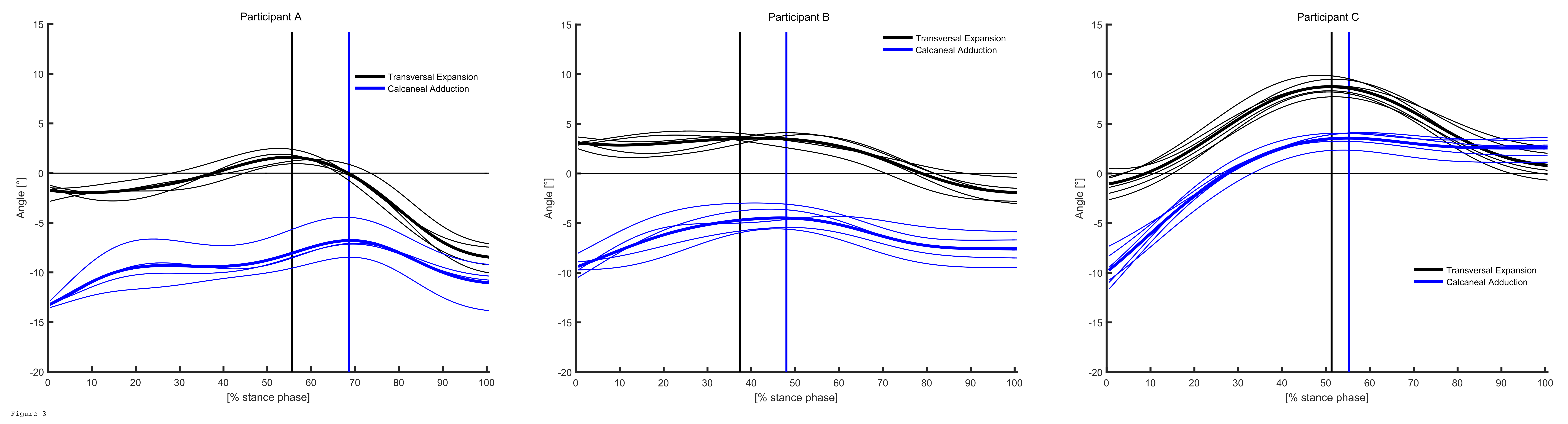

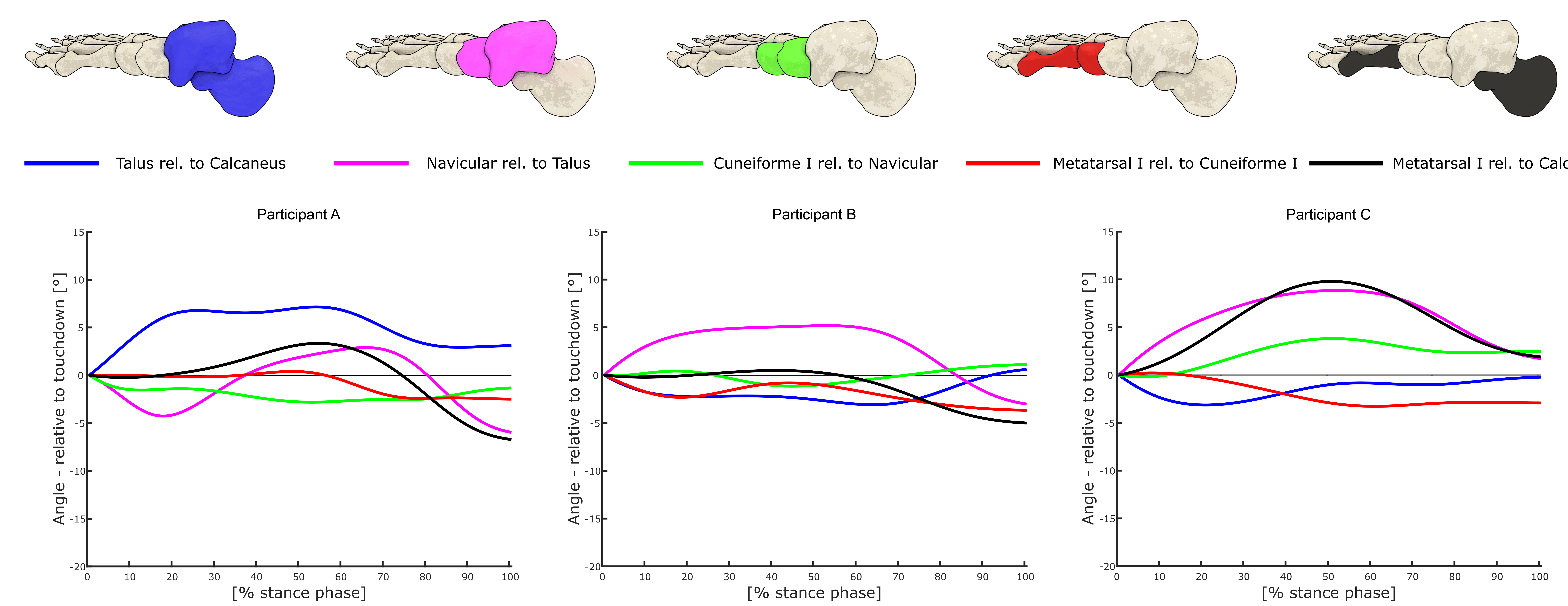

Cuneiforme I rel. to Navicular

Metatarsal I rel. to Cuneiforme I $\longrightarrow$ Metatarsal I rel. to Calcaneus
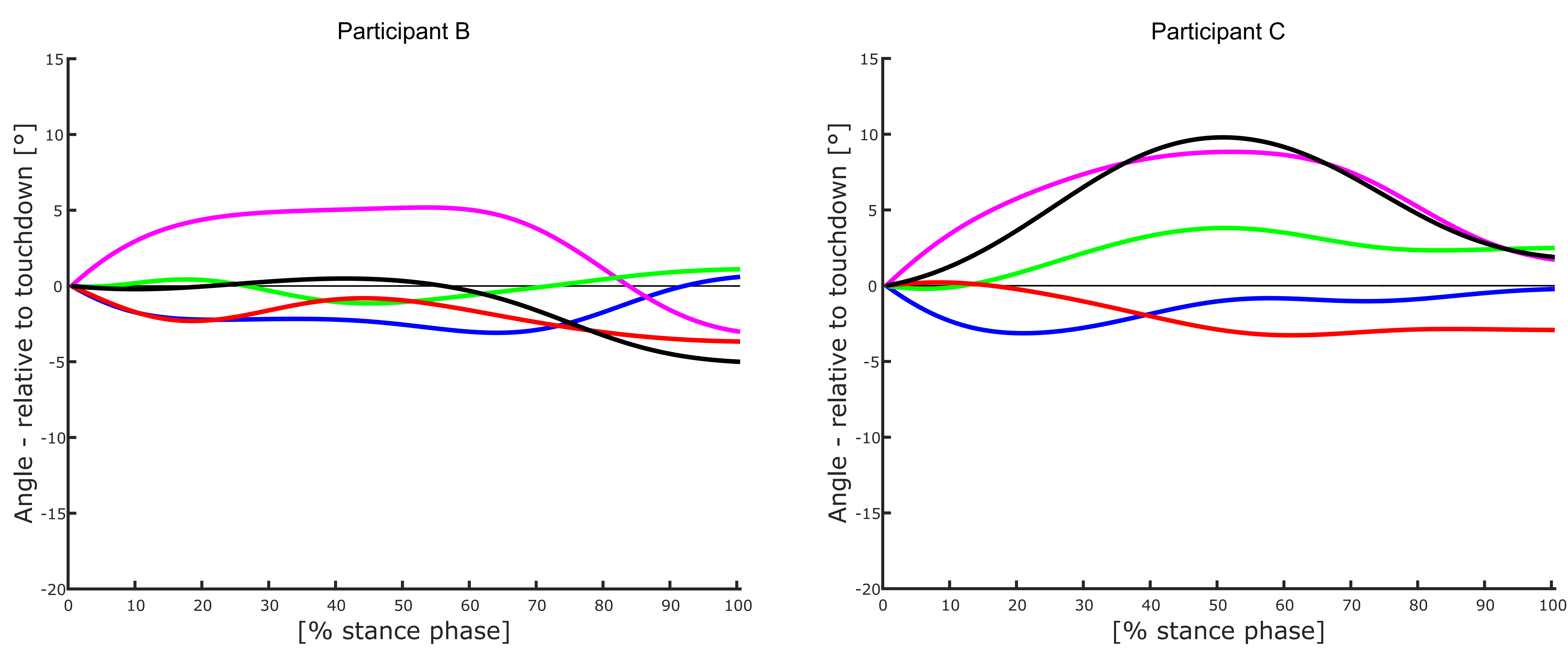
Eigure 4 